\section{Inadequações na ingestão dietética de micronutrientes por homens e mulheres residentes no Sul do Brasil: Estudo EpiFloripa Adultos 2012}

\author{
Inadequate dietary micronutrient intake in men \\ and women in southern Brazil: the EpiFloripa \\ Adults Study, 2012
}

Inadecuaciones en la ingestión dietética de micronutrientes por hombres y mujeres residentes en el sur de Brasil: Estudio EpiFloripa

Adultos 2012

\begin{abstract}
Resumo
O objetivo do estudo foi estimar a ingestão dietética de micronutrientes por adultos em um município do Sul do Brasil segundo sexo e idade, assim como identificar a prevalência de ingestão inadequada de micronutrientes segundo sexo. Trata-se de um estudo transversal, de base populacional, com 1.222 adultos de 22 a 63 anos, residentes em Florianópolis, Santa Catarina, Brasil. Os dados sobre a ingestão alimentar foram obtidos pela aplicação de dois recordatórios de 24 horas (um no total da amostra e outro em subamostra de $40 \%$ dos entrevistados), sendo a ingestão de micronutrientes e as prevalências de inadequação na ingestão estimadas conforme recomendações do National Research Council e do Institute of Medicine. Prevalências elevadas de inadequação para toda a amostra foram vistas para as vitaminas A, C, D e E (variação de 52\%-100\%). A ingestão de cálcio e ferro foi a mais inadequada entre as mulheres (87,3\% e 13,7\%, respectivamente), e a de zinco entre os homens (25,1\%). Adultos de Florianópolis apresentaram prevalências elevadas de inadequação na ingestão de micronutrientes essenciais.
\end{abstract}

Ingestão de Alimentos; Micronutrientes; Adulto
Francieli Cembranel 1 David Alejandro González-Chica 2 Eleonora d'Orsi 1



1 Centro de Ciências da Saúde, Universidade Federal de Santa Catarina, Florianópolis, Brasil. 2 Adelaide Medical School, The University of Adelaide, Adelaide, Australia. 


\section{Introdução}

Seguindo a tendência mundial, os padrões de consumo alimentar no Brasil sofreram intensas e rápidas modificações nas últimas quatro décadas. Nesse espaço de tempo, houve não apenas redução na participação de alimentos tradicionais da dieta como cereais, leguminosas e hortaliças, como também aumento da ingestão de preparações com elevada concentração de açúcar, sódio e gordura saturada 1,2 .

Como resultado, inquéritos epidemiológicos têm mostrado repercussões negativas de todas essas mudanças à saúde da população, sobretudo devido à perda do teor nutricional de micronutrientes pela dieta 1,2,3,4,5. Evidências consistentes mostram que baixos níveis plasmáticos de zinco, vitaminas A e C estão associados com o estado nutricional de obesidade 3 . Do mesmo modo, a baixa ingestão de vitamina D e cálcio tem sido associada ao aumento da prevalência de doenças cardiovasculares e osteoporose 4 . Semelhantemente, a maioria dos casos de anemia é atribuída à deficiência de ferro 5 .

Diante disso, investigar aspectos específicos da ingestão alimentar em nível populacional, como vitaminas e minerais, pode ser de particular importância, uma vez que essas evidências podem contribuir não só no direcionamento de políticas públicas voltadas a melhorar o estado nutricional em nível populacional, mas sobretudo para a prevenção de doenças crônicas. A maior parte dos estudos sobre essa temática é oriunda de países europeus 6 e dos Estados Unidos 7 , sendo as evidências a esse respeito limitadas em países de renda média como o Brasil 8 . Assim, o objetivo deste trabalho foi estimar a ingestão dietética de micronutrientes por adultos residentes em um município do Sul do Brasil segundo sexo e idade, assim como identificar a prevalência de ingestão inadequada de micronutrientes segundo sexo.

\section{Métodos}

Análise transversal da segunda onda do estudo longitudinal de base populacional EpiFloripa Adultos, composta por amostra de 1.222 indivíduos com idades entre 22-63 anos, residentes na área urbana de Florianópolis, Santa Catarina, Região Sul do Brasil. Maiores detalhes sobre os aspectos metodológicos do EpiFloripa Adultos podem ser consultados em publicação prévia 9 .
Os dados sobre a ingestão dietética dos participantes foram obtidos mediante a aplicação de dois recordatórios de 24 horas (R24h). O primeiro R24h foi aplicado no total da amostra durante a entrevista domiciliar, e o segundo por telefone em uma subamostra de $40 \%$ desses participantes 10 . Desse modo, foi possível alcançar a variabilidade da ingestão e proceder ao ajuste das variáveis dietéticas pela variabilidade intraindividual e interindividual 11,12,13.

Informações sobre as variáveis demográficas (sexo, idade, estado civil) e socioeconômicas (renda familiar mensal per capita e escolaridade) também foram obtidas dos participantes.

\section{Processamento e análise dos dados}

As informações obtidas dos dois R24h foram inseridas no software Nutrition Data System for Research (NDSR) (Nutrition Coordinating Center, University of Minnesota, Minneapolis, Estados Unidos), sendo que os alimentos brasileiros que não constavam na base de dados do programa tiveram seus valores nutricionais inseridos de acordo com dados nacionais 14 . Desse modo, obtiveram-se do NDSR informações nutricionais relativas à ingestão dietética de cálcio, ferro, zinco, vitaminas A, C, D e E para cada participante. Seguindo as recomendações do National Research Council e do Institute of Medicine (IoM), antes das análises todas essas variáveis foram ajustadas pela variabilidade intraindividual e interindividual por meio da fórmula: ingesta ajustada: [(média do sujeito-média do grupo) $\mathrm{x}$ (desvio padrão interpessoal/desvio padrão observado)] + média grupo 11,12,13 .

A análise dos dados foi conduzida no software Stata versão 13.0 (StataCorp LP, College Station, Estados Unidos). Para a descrição das características da população do estudo foram consideradas as frequências absolutas e relativas. Para a descrição das variáveis contínuas representativas da ingestão de micronutrientes, os dados foram apresentados em mediana e intervalo interquartil.

Para avaliar as prevalências de inadequação da ingestão de cálcio, zinco, vitaminas A, C, D e E foram considerados como ponto de corte os valores da Estimated Average Requirement (EAR) segundo o sexo e a faixa etária, empregando-se a fórmula: escore-Z: (média ajustada - EAR)/ desvio padrão da distribuição ajustada 13,15. Na avaliação da inadequação da ingestão de ferro, por sua vez, foi empregada a abordagem probabilística manualmente determinada 13 . 
A ingestão alimentar mediana de micronutrientes segundo faixas etárias também foi avaliada 13,15 .

Em todas as análises foram utilizados pesos amostrais, combinando o desenho da amostra na linha de base, a probabilidade de localização dos participantes em 2012 em cada setor censitário, e a população estimada de Florianópolis em 2012. O valor de significância estatística adotado foi de $\mathrm{p} \leq 0,05$.

\section{Aspectos éticos}

O EpiFloripa Adultos foi aprovado em ambas as ondas pelo Comitê de Ética em Pesquisa com Seres Humanos da Universidade Federal de Santa Catarina (processos 351/2008 e 1772/2011).

\section{Resultados}

Na linha de base do EpiFloripa Adultos foram entrevistados 1.720 indivíduos. Desses, foram localizados e entrevistados novamente 1.222 adultos em 2012. Nas duas ondas houve maior proporção de mulheres e de pessoas casadas/morando com companheiro, e não houve diferenças nos percentuais de acompanhamento conforme a escolaridade. Contudo, em 2012 houve maior proporção de perdas de participantes no terço inferior de renda e mais jovens (20-29 anos). Mesmo assim, $60 \%$ dos indivíduos nessas categorias foram localizados (Tabela 1).

Quanto à ingestão dietética (Tabela 2) para as vitaminas A e C, as medianas de ingestão foram maiores entre as mulheres, enquanto a de cálcio, ferro, zinco e das vitaminas D e E foi superior nos homens $(\mathrm{p}<0,001)$. Em relação às inadequações, tanto homens quanto mulheres apresentaram prevalências de ingestão inadequada elevadas, oscilando entre $52 \%-100 \%$ para as vitaminas A, C, D e E. Comparadas aos homens, as mulheres apresentaram maior prevalência de inadequação na ingestão de cálcio (87,3\% entre aquelas de 5163 anos) e de ferro (13,7\% entre as de 20-50 anos). Os homens, por sua vez, apresentaram maior porcentual de ingestão inadequada de zinco e das vitaminas A e C.

A ingestão dietética de micronutrientes ainda foi analisada segundo a idade (Figura 1). Com exceção do ferro e do zinco, para os demais micronutrientes, indivíduos de ambos os sexos apresentaram de modo geral mediana de ingestão abaixo dos valores da EAR e com pouca variação entre as faixas etárias. A maior variação foi observada para a vitamina $C$, que aumentou com a idade entre as mulheres $(\mathrm{p}<0,001)$.

\section{Discussão}

Com base nos resultados encontrados, foi possível observar que adultos de Florianópolis, de modo geral, apresentaram prevalências elevadas de inadequação na ingestão dietética de micronutrientes. Além disso, a ingestão dos micronutrientes avaliados não apresentou alterações importantes com a idade, com exceção da vitamina $\mathrm{C}$, que foi maior entre participantes de idades mais avançadas, sobretudo entre as mulheres.

Esses achados corroboram resultados prévios tanto na literatura internacional quanto nacional. Estudos com dados da Alemanha, Estados Unidos, Reino Unido e México 3,6,7, além do próprio Brasil 8, mostram prevalências de consumo inadequado de micronutrientes igualmente elevadas, sobretudo para as vitaminas A, C, D, E e cálcio. Considerando o papel protetor desses micronutrientes na etiologia e progressão de condições crônicas, como doenças cardiovasculares, neoplasias, diabetes e obesidade, doenças com alta prevalência entre a população adulta mundial na atualidade 1,2,3,4, sugere-se a adoção de intervenções para a redução dessas inadequações, como por exemplo, incentivo ao aumento da ingestão de frutas cítricas e hortaliças, fontes das vitaminas A, C e E. Sugerimos ainda que tais estratégias incluam incentivo ao aumento da ingestão de alimentos fonte de cálcio, como leite e derivados, especialmente entre mulheres com mais de 50 anos, e de ferro entre aquelas em idade reprodutiva, tendo em vista a gravidade de ambas as deficiências para a saúde.

$\mathrm{O}$ incentivo à ingestão de alimentos como fígado e ovos e à exposição solar adequada, também se faz necessário para a redução da deficiência de vitamina D. Ressaltamos, contudo, que não recomendamos que o aumento da ingestão de vitaminas e minerais se baseie na suplementação, considerando que tal estratégia pode desestimular a adoção de hábitos alimentares saudáveis 8 . Assim, ênfase deve ser dada na formulação de políticas de incentivo à adoção de uma alimentação saudável, incluindo políticas de segurança alimentar, que possam contribuir para reverter esse cenário e ainda atuar na prevenção de doenças crônicas e outros agravos decorrentes da baixa ingestão de micronutrientes. 
Cembranel F et al.

Tabela 1

Tabela comparativa das características socioeconômicas e demográficas da população pesquisada. Estudo EpiFloripa Adultos 2009 e 2012. Florianópolis, Santa Catarina, Brasil, 2012.

\begin{tabular}{|c|c|c|c|c|}
\hline \multirow[t]{2}{*}{ Variáveis } & $\begin{array}{l}\text { Amostra na linha de base } \\
\qquad \begin{array}{c}(2009) \\
{[n=1.720]}\end{array}\end{array}$ & $\begin{array}{c}\text { Amostra } \\
\text { da 2a onda } \\
\text { (2012) } \\
{[n=1.222]}\end{array}$ & $\begin{array}{l}\text { Entrevistados em } 2012 \text { em relação aos } \\
\text { entrevistados na linha de base (2009) }\end{array}$ & Valor de $p$ * \\
\hline & n (\%) & n (\%) & $\%$ & \\
\hline \multicolumn{5}{|l|}{ Sexo } \\
\hline Masculino & $761(44,2)$ & $522(48,2)$ & 68,6 & 0,046 \\
\hline Feminino & $959(55,8)$ & $700(51,8)$ & 73,0 & \\
\hline \multicolumn{5}{|l|}{ Idade (anos) } \\
\hline $20-29$ & $540(31,4)$ & $328(26,8)$ & 60,7 & $<0,001$ \\
\hline $30-39$ & $392(22,8)$ & $277(22,7)$ & 70,7 & \\
\hline $40-49$ & $438(25,5)$ & $345(28,2)$ & 78,8 & \\
\hline $50-63$ & $350(20,3)$ & $272(22,3)$ & 77,7 & \\
\hline \multicolumn{5}{|l|}{ Estado civil } \\
\hline Casado/Morando com companheiro & $1043(60,6)$ & $779(63,8)$ & 74,7 & $<0,001$ \\
\hline Solteiro/Divorciado/Viúvo & $677(39,4)$ & $443(36,2)$ & 65,4 & \\
\hline \multicolumn{5}{|l|}{ Escolaridade (anos de estudos) } \\
\hline $0-4$ & $158(9,2)$ & $108(8,9)$ & 68,4 & 0,217 \\
\hline $5-8$ & $253(14,7)$ & $174(14,3)$ & 68,8 & \\
\hline $9-11$ & $568(33,1)$ & $394(32,3)$ & 69,4 & \\
\hline 12 ou mais & $737(43,0)$ & $543(44,5)$ & 73,7 & \\
\hline \multicolumn{5}{|l|}{ Renda familiar per capita } \\
\hline 1ㅇ terço (inferior) & $564(33,5)$ & $382(31,7)$ & 67,7 & 0,040 \\
\hline 2o terço (intermediário) & $562(33,4)$ & $409(33,9)$ & 72,8 & \\
\hline 3o terço (superior) & $559(33,1)$ & $415(34,4)$ & 74,2 & \\
\hline
\end{tabular}

* Valor de p do teste de qui-quadrado com correção de Rao-Scott comparando entrevistados em 2012 em relação aos não entrevistados. 
Tabela 2

Mediana, intervalo interquartil e prevalência de inadequação na ingestão dietética de micronutrientes pela população do estudo, estratificada segundo os sexos. Florianópolis, Santa Catarina, Brasil, 2012.

\begin{tabular}{|c|c|c|c|c|c|c|c|}
\hline \multirow[t]{2}{*}{ Variáveis } & \multicolumn{2}{|c|}{ Amostra } & \multicolumn{2}{|c|}{ Homens } & \multicolumn{2}{|c|}{ Mulheres } & \multirow[t]{2}{*}{ Valor de $p$ * } \\
\hline & Mediana & p25-p75 & Mediana & P25-P75 & Mediana & P25-P75 & \\
\hline Vitamina A ( $\mu g R e)$ & 289,4 & $229,6-359,5$ & 287,1 & $222,8-366,3$ & 291,1 & $233,7-353,8$ & $<0,001$ \\
\hline Vitamina C (mg) & 46,8 & $26,0-80,5$ & 45,7 & $25,6-79,0$ & 48,0 & $26,4-81,6$ & $<0,001$ \\
\hline Vitamina D $(\mu \mathrm{g})$ & 3,4 & $2,5-4,4$ & 3,5 & $2,6-4,6$ & 3,3 & $2,4-4,2$ & $<0,001$ \\
\hline Vitamina $E(m g)$ & 5,4 & $4,4-6,6$ & 5,7 & $4,6-6,8$ & 5,2 & $4,2-6,3$ & $<0,001$ \\
\hline Cálcio (mg) & 758,3 & $597,0-928,5$ & 795,5 & $646,3-971,5$ & 716,5 & $570,0-886,6$ & $<0,001$ \\
\hline Ferro (mg) & 11,6 & $10,7-12,6$ & 12,1 & $11,4-13,1$ & 11,1 & $10,3-11,9$ & $<0,001$ \\
\hline Zinco (mg) & 10,8 & $9,7-11,6$ & 10,8 & $9,4-12,2$ & 9,6 & $8,4-10,9$ & $<0,001$ \\
\hline Prevalência de inadequação & & $\%$ ** & EAR & $\%$ & EAR & $\%$ & Valor de $p^{\star \star \star \star}$ \\
\hline Vitamina A ( $\mu g R e)$ & & 98,0 & $625 \mu \mathrm{g}$ & 98,5 & $500 \mu \mathrm{g}$ & 97,6 & $<0,001$ \\
\hline Vitamina C & & 57,0 & $75 \mathrm{mg}$ & 63,9 & $60 \mathrm{mg}$ & 52,0 & $<0,001$ \\
\hline Vitamina D & & 100,0 & $10 \mu \mathrm{g}$ & 100,0 & $10 \mu \mathrm{g}$ & 100,0 & 0,204 \\
\hline Vitamina E & & 98,9 & $12 \mathrm{mg}$ & 98,1 & $12 \mathrm{mg}$ & 99,5 & $<0,001$ \\
\hline Cálcio & & 59,1 & & & & & \\
\hline $20-50$ anos & & & $800 \mathrm{mg} \#$ & 46,6 & $800 \mathrm{mg}$ & 58,8 & $<0,001 \# \#$ \\
\hline $51-63$ anos & & & & & $1000 \mathrm{mg}$ & 87,3 & \\
\hline Ferro & & 0,1 & & & & & \\
\hline $20-50$ anos & & & $6 \mathrm{mg} \#$ & $0,0 \# \#$ & $8,1 \mathrm{mg}$ & 13,7 \#\#\# & $0,025 \# \#$ \\
\hline $51-63$ anos & & & & & $5 \mathrm{mg}$ & 0,0 \#\#\# & \\
\hline Zinco & & 18,1 & $9,4 \mathrm{mg}$ & 25,1 & $6,8 \mathrm{mg}$ & 13,0 & $<0,001$ \\
\hline
\end{tabular}

\%: percentual de ingestão inadequada do nutriente; EAR: Estimated Average Requirement;

* Valor de $\mathrm{p}$ do teste de Mann-Whitney;

** A prevalência geral de inadequação da amostra foi obtida por uma média ponderada das prevalências de inadequação encontradas em homens e muheres;

*** Valor de $p$ do teste de qui-quadrado com correção de Rao-Scott;

\# Recomendação da EAR para homens nas duas faixas etárias;

\#\# Valor de p da prevalência de inadequação em homens comparado com a prevalência combinada de inadequação em mulheres das duas faixas etárias;

\#\#\# Percentual de inadequação obtido pela abordagem probabilística manualmente determinada 13. 
Figura 1

Ingestão alimentar mediana de micronutrientes pela população do estudo, segundo os sexos e faixas etárias. Florianópolis, Santa Catarina, Brasil, 2012.

a) Cálcio

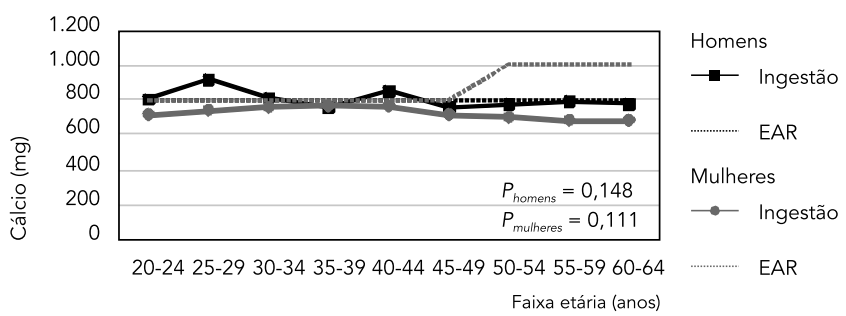

c) Vitamina A

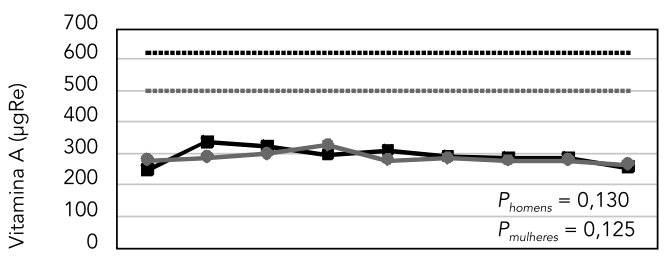

20-24 25-29 30-34 35-39 40-44 45-49 50-54 55-59 60-64

Faixa etária (anos)

e) Vitamina C

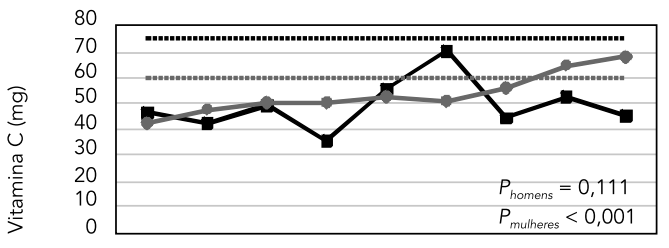

20-24 25-29 30-34 35-39 40-44 45-49 50-54 55-59 60-64

Faixa etária (anos)

g) Zinco

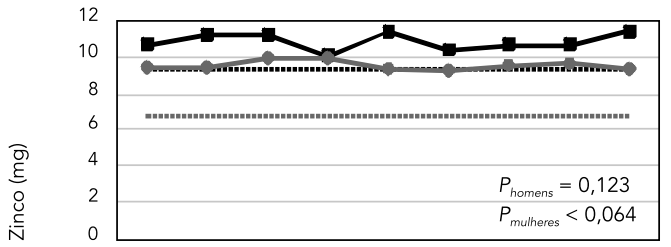

20-24 25-29 30-34 35-39 40-44 45-49 50-54 55-59 60-64

Faixa etária (anos)

$\longrightarrow$ Ingestão b) Ferro

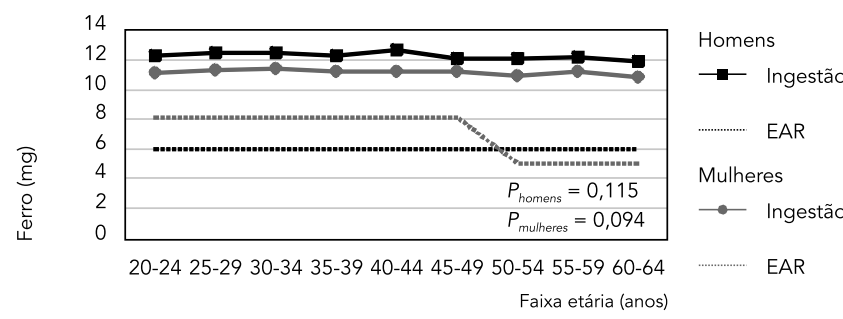

d) Vitamina $D$

Homens

- Ingestão

-......... EAR

Mulheres

$\longrightarrow$ Ingestão

............... EAR

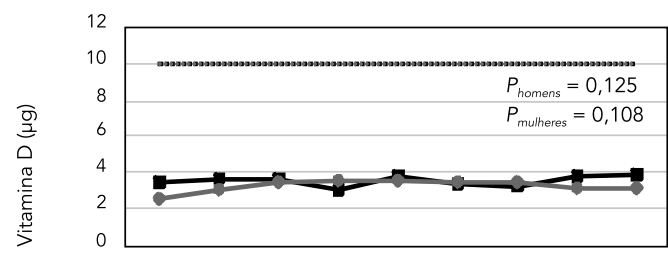

20-24 25-29 30-34 35-39 40-44 45-49 50-54 55-59 60-64

Faixa etária (anos)

f) Vitamina $E$

Homens

- Ingestão

………... EAR

Mulheres

EAR

के

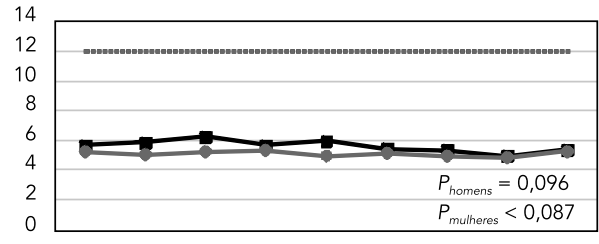

Homen

- Ingestão

………. EAR

Mulheres

$\longrightarrow$ Ingestão

20-24 25-29 30-34 35-39 40-44 45-49 50-54 55-59 60-64

Faixa etária (anos)
Homens

- Ingestão

EAR

Mulheres

$\because$ Ingestão

EAR

EAR: Estimated Average Requirement; P: valor de p do teste de Kruskal-Wallis. 


\section{Colaboradores}

F. Cembranel, D. A. González-Chica e E. d'Orsi participaram igualmente na concepção do trabalho, análise e interpretação dos dados, redação do artigo e na aprovação da versão final submetida. São responsáveis por todos os aspectos do trabalho, na garantia da exatidão e integridade de qualquer parte da obra.

\section{Agradecimentos}

Agradecemos aos participantes do estudo, à Capes pela bolsa de doutorado concedida a F. Cembranel, e ao CNPq pelo financiamento do Projeto EpiFloripa Adultos 2009 e 2012 Estudo Epidemiológico das Condições de Saúde dos Adultos do Município de Florianópolis, Santa Catarina, Brasil (editais universais no 485327/2007-4 e oo 477061/2010-9), do qual o presente artigo faz parte.

\section{Referências}

1. Monteiro CA, Levy RB, Claro RM, Castro IRR, Cannon G. Increasing consumption of ultraprocessed foods and likely impact on human health: evidence from Brazil. Public Health Nutr 2011; 14:5-13.

2. Popkin BM, Adair LS, Ng SW. Global nutrition transition and the pandemic of obesity in developing countries. Nutr Rev 2012; 70:3-21.

3. Garcia OP, Ronquillo D, Caamaño MC, Camacho M, Long KZ, Rosado JL. Zinc, vitamin A, and vitamin C status are associated with leptin concentrations and obesity in Mexican women: results from a cross-sectional study. Nutr Metab (Lond) 2012; 9:59.

4. Carvalho LS, Sposito AC. Vitamin D for the prevention of cardiovascular disease: are we ready for that? Atherosclerosis 2015; 241:729-40.

5. Mujica-Coopman MF, Brito A, López de Romaña D, Coris H, Olivares M. Prevalence of Anemia in Latin America and the Caribbean. Food Nutr Bull 2015; 36(2 Suppl):S119-28.

6. Troesch B, Hoeft B, McBurney M, Eggersdorfer M, Weber P. Dietary surveys indicate vitamin intakes below recommendations are common in representative Western countries. Br J Nutr 2012; 108:692-8.

7. Agarwal S, Reider C, Brooks JR, Fulgoni VL. Comparison of prevalence of inadequate nutrient intake based on body weight status of adults in the United States: an analysis of NHANES 2001-2008. J Am Coll Nutr 2015; 34:126-34.

8. Araujo MC, Bezerra IN, Barbosa F dos S, Junger WL, Yokoo EM, Pereira RA, et al. Macronutrient consumption and inadequate micronutrient intake in adults. Rev Saúde Pública 2013; 47 Suppl 1:S177-89.
9. Boing AC, Peres KG, Boing AF, Hallal PC, Silva NN, Peres MA. EpiFloripa Health Survey: the methodological and operational aspects behind the scenes. Rev Bras Epidemiol 2014; 17:147-62.

10. Verly-Jr E, Castro MA, Fisberg RM, Marchioni DM. Precision of usual food intake estimates according to the percentage of individuals with a second dietary measurement. J Acad Nutr Diet 2012; 112:1015-20.

11. Willett WC, Howe GR, Kushi LH. Adjustment for total energy intake in epidemiologic studies. Am J Clin Nutr 1997; 65(4 Suppl):S1220-8.

12. Dodd KW, Guenther PM, Freedman LS, Subar AF, Kipnis V, Midthune D, et al. Statistical methods for estimating usual intake of nutrients and foods: a review of the theory. J Am Diet Assoc 2006; 106:1640-50.

13. Otten JJ, Hellwig JP, Meyers LD, editors. Dietary reference intakes: the essential guide to nutrient requirements. Washington DC: National Academy Press; 2006.

14. Núcleo de Estudos e Pesquisas em Alimentação, Universidade Estadual de Campinas. Tabela brasileira de composição de alimentos (TACO), 2011. http://www.unicamp.br/nepa/taco/tabela.php?a tivo=tabela (acessado em 01/Mar/2013).

15. Institute of Medicine. Dietary reference intakes for calcium and vitamin d. Washington DC: National Academy Press; 2011. 


\section{Abstract}

This study aimed to estimate dietary intake of micronutrients among adults in a city in Southern Brazil according to sex and age, and to measure the prevalence of inadequate dietary micronutrient intake according to sex. This is a cross-sectional, population-based study with 1,222 adults 22 to 63 years of age living in Florianópolis, Santa Catarina State, Brazil. Data on dietary intake were obtained from two 24-hour recalls (one in the total sample and the other in a subsample of $40 \%$ of the research subjects). Micronutrient intake and prevalence rates for inadequate intake were estimated according to the guidelines of the National Research Council and Institute of Medicine. High prevalence rates of inadequate intake in the entire sample were seen for vitamins $A, C, D$, and $E$ (range, 52\%-100\%). Calcium and iron intake were more inadequate in women (87.3\% and $13.7 \%$, respectively), as was zinc in men (25.1\%). Adults in Florianópolis, showed high prevalence rates of inadequate intake of essential micronutrients.

Eating; Micronutrients; Adult

\section{Resumen}

El objetivo del estudio fue estimar la ingestión dietética de micronutrientes por parte de adultos en un municipio del sur de Brasil, según sexo y edad, así como identificar la prevalencia de ingestión inadecuada de micronutrientes según sexo. Se trata de un estudio transversal, de base poblacional, con 1.222 adultos de 22 a 63 años, residentes en Florianópolis, Santa Catarina, Brasil. Los datos sobre la ingestión alimentaria se obtuvieron mediante la aplicación de dos recordatorios de 24hrs. (uno en el total de la muestra y otro en una submuestra de un $40 \%$ de los entrevistados), siendo estimadas la ingestión de micronutrientes y las prevalencias de inadecuación en la ingestión conforme las recomendaciones del National Research Council y del Institute of Medicine. Prevalencias elevadas de inadecuación para toda la muestra fueron observadas en las vitaminas A, C, D y E (variación de un 52\%-100\%). La ingestión de hierro y calcio fue la más inadecuada entre las mujeres $(87,3 \%$ y 13,7\%, respectivamente), y la de zinc entre los hombres (25,1\%). Los adultos de Florianópolis presentaron prevalencias elevadas de inadecuación en la ingestión de micronutrientes esenciales.

Ingestión de Alimentos; Micronutrientes; Adulto
Recebido em 24/Mar/2016

Versão final reapresentada em 04/Mai/2016 Aprovado em 08/Jul/2016 